Revue européenne des sciences sociales

European Journal of Social Sciences

XL-124 | 2002

Histoire, philosophie et sociologie des sciences

\title{
Prix relatifs et structures des marchés. Dialogue hors du temps avec Luigi Solari
}

Emilio Fontela

\section{(2) OpenEdition}

12 Journals

Édition électronique

URL : https://journals.openedition.org/ress/597

DOI : $10.4000 /$ ress. 597

ISSN : 1663-4446

Éditeur

Librairie Droz

Édition imprimée

Date de publication : 1 août 2002

Pagination : 319-331

ISBN : 2-600-00806-3

ISSN : 0048-8046

Référence électronique

Emilio Fontela, "Prix relatifs et structures des marchés. Dialogue hors du temps avec Luigi Solari », Revue européenne des sciences sociales [En ligne], XL-124 | 2002, mis en ligne le 01 décembre 2009, consulté le 21 septembre 2021. URL : http://journals.openedition.org/ress/597 ; DOI : https://doi.org/ $10.4000 /$ ress.597

(c) Librairie Droz 
Emilio FONTELA

\section{PRIX RELATIFS ET STRUCTURES DES MARCHÉS DIALOGUE HORS DU TEMPS AVEC LUIGI SOLARI*}

En janvier 1971 eut lieu à Genève la Cinquième Conférence Internationale sur les Techniques Input-Output; Luigi Solari, André Duval et moi-même présentâmes une communication sur les effets de contraintes de production dans un modèle ouvert de Leontief; ultérieurement nous publiâmes une version française de cette communication (Fontela, Solari, Duval, 1971) et la version définitive en anglais (Fontela, Solari, Duval, 1972). Trente ans après il m'a semblé intéressant, à l'occasion de la Conférence Solari 2000, de revenir sur ce travail et de le replacer dans le contexte actuel du développement de la mésoéconomie.

En premier lieu, il s'agira donc de décrire le contenu de cette publication, et on ne pourra mieux le faire qu'en reproduisant le résumé, dont j'ai souvenir qu'il avait été écrit par Luigi Solari lui-même:

La présente étude vise à analyser l' influence de contraintes de production et de consommation sur les principales variables d' un système économique initialement à l'équilibre. Dans le contexte de la politique économique, il s'agit notamment d'étudier les restructurations de prix et de valeurs ajoutées rendues nécessaires par d'éventuelles réductions de la capacité de production de certains secteurs.

L'étude avait donc un but précis : celui d'apporter des éléments pour une prise de décision en cas de chocs externes, caractérisés dans ce cas par des contraintes sur la production et la consommation. L'approche trouva sa pleine justification deux années plus tard, en 1973, lorsque les disponibilités de pétrole furent soudainement restreintes par le cartel de l'OPEP.

Le modèle adopté utilisait deux instruments traditionnels de l'économétrie appliquée: l'analyse input-output et les systèmes de fonction de consommation. Ces deux instruments opératoires ont fourni les premières lignes de recherche du Département d'Econométrie, aboutissant à plusieurs thèses de doctorat. Par exemple, celles de Baranzini (1978), Antille (1978), McNeill (1978), Juillard (1993) ou Garau (1996) dans le domaine de l'input-output, et celles du regretté Rossier (1974), de Carlevaro (1975) ou de Snella (1976) dans le domaine des fonctions de consommation.

Pour être plus précis, il s'agissait de compléter le modèle ouvert de Leontief, par l'adjonction d'un Système Linéaire de Dépenses (SLD) rendant la demande finale sensible aux variations des prix et des revenus. A la lumière des développements récents de l'économétrie appliquée, on peut dire qu'il s'agissait d'élaborer

* Conférence Luigi Solari 2000 prononcée à l’Université de Genève le 14 novembre 2000. 
un ancêtre linéaire et avec rendements constants des modèles d'équilibre général calculables, dans lesquels la fonction de production de Leontief avec coefficients techniques fixes est remplacée par des fonctions telles que celles de Jorgensen, ayant des coefficients techniques élastiques aux prix et à la production, et le SLD est remplacé à son tour par des systèmes plus proches d'un modèle idéal de fonctions de demande de Deaton.

Le problème posé par les contraintes de production avait été analysé par Stone (1961) à l'aide d'un modèle input-output ouvert établissant une partition entre secteurs contraints et secteurs sans contraintes. Dans ce contexte Stone avait pu préciser que, du fait des relations intersectorielles, il existe toujours une relation entre la demande finale des secteurs contraints $\left(f_{r}\right)$ et celle des secteurs sans contraintes $\left(f_{s}\right)$ telle que:

$$
f_{r}=H q_{r}-G f_{s}
$$

ou $G$ et $H$ sont des matrices de coefficients dérivés matriciellement de la matrice des coefficients techniques input-output, notée traditionnellement par $A$, et $q_{r}$ est le vecteur des contraintes sur la production.

La relation (1) décrit le fait que la consommation finale de biens soumis à des contraintes de production ne va pas dépendre uniquement de ces dernières et qu'il existe aussi des possibilités de manoeuvre par modification de la demande des biens non contraints. Il s'agit donc bien de la formalisation de l'argument de ceux qui, au moment de la crise du pétrole, entendaient qu'il ne s'agissait pas uniquement de consommer moins d'essence, mais qu'il était nécessaire de modifier les styles de vie, et de réduire toutes les consommations qui indirectement contenaient aussi de l'énergie et du pétrole.

Stone était conscient que cette démonstration simple et linéaire de l'importance de l'interdépendance mésoéconomique était insuffisante; encore fallait-il préciser quels étaient les biens dont on devait réduire la consommation en maintenant le niveau de bien-être le plus élevé possible, compte tenu des circonstances. C'est cette décision relative à la structure de la réduction de la consommation que nous avions essayé d'analyser dans notre étude, sur la base d'un système linéaire de dépenses (SLD) conforme à la théorie des choix du consommateur (Solari, 1971).

A cette époque, la portée opératoire du SLD avait été bien établie, et le choix se porta tout naturellement sur ce type de fonctions déjà utilisées par Klein et par Stone.

Le programme de maximisation du bien-être que nous avions adopté était très simple, et peut être résumé comme suit: en notant par $\bar{f}, \bar{q}$ et $\bar{p}$ les valeurs d'équilibre initiales de la demande, de la production et des prix respectivement, et par $f$, $q$ et $p$ les valeurs correspondantes de la solution, on pose:

$$
\begin{gathered}
\min _{f}(f-\bar{f})^{\prime} W(f-\bar{f}) \\
f \geq \Upsilon \\
q \leq \hat{\lambda}_{\bar{q}} \\
p^{\prime} f=\bar{p}^{\prime} f
\end{gathered}
$$




$$
\begin{gathered}
f=\Upsilon+\hat{p}^{-1} \beta p^{\prime}[f-\Upsilon] \\
v=\left(I-A^{\prime}\right) p
\end{gathered}
$$

D'après cette spécification, il s'agissait donc, en respectant des contraintes sur la consommation et la production, de minimiser les variations de la demande finale, mesurées par l'indicateur collectif (2) dans lequel W est une matrice de pondération ( $W=I$ si toutes les différences sont équivalentes).

Les contraintes de la relation (3) spécifient que la demande d'un bien ne peut être inférieure à la consommation obligée $\gamma$ (calculée par l'estimation du SLD en même temps que les coefficients $\beta$ qui ventilent par secteurs les consommations excédentaires); celles de la relation (4) que la production ne pourra pas être supérieure à la capacité (définie par un coefficient $\lambda_{i}$ de la production d'équilibre de chaque bien $i$ ).

Quant aux relations (6), elles reproduisent l'aspect demande du modèle d'après les fonctions de consommation du SLD qui ont été retenues. Avec la relation (5) elles déterminent ainsi les nouveaux prix relatifs $p$. Les nouvelles valeurs ajoutées $v$ s'en déduisent par la relation (7).

Sur le plan empirique, ce modèle qui est appelé dans la suite de cette communication le modèle FSD, fut appliqué avec des données italiennes pour calculer les effets de contraintes sur les productions d'électricité, de produits pétrochimiques et d'habillement qui réduisaient l'offre de ces secteurs de $20 \%$ conduisant à des réductions de l'ordre de $50 \%$ pour la demande finale des deux premiers secteurs et de $20 \%$ pour l'habillement, secteur dans lequel la production est plus directement reliée à la consommation finale. Pour arriver à ce résultat les prix relatifs de l'électricité augmentent de $150 \%$, ceux de la pétrochimie de $90 \%$ et ceux de l'habillement de $50 \%$. Les productions, les demandes finales et les prix de tous les secteurs sont modifiés en conséquence du fait des interdépendances du système. La nouvelle structure des prix relatifs modifie sensiblement les valeurs ajoutées sectorielles, générant des surplus positifs ou négatifs qui affecteront les rémunérations des travailleurs, des capitalistes ou des administrations publiques par la voie des taxes indirectes ou des subventions dans le dernier cas.

Nous concluions alors en exprimant l'idée qu'une politique fiscale éclairée devrait permettre une maximisation du bien-être collectif au sens du critère de départ de moindre distorsion de la consommation préalable à l'apparition des contraintes. Dans une certaine mesure, c'est ce qui a été fait dans le cas concret de la crise pétrolière lorsque, dans tous les pays consommateurs, les taxes indirectes sur les carburants liquides ont été augmentées fortement.

Sans doute ce modèle, avec des légères modifications, aurait pu être appliqué pour étudier les effets sectoriels d'autres chocs externes sur les productions, sur les demandes finales, sur les prix ou sur les valeurs ajoutées mais toujours dans un cadre statique, avec des structures technologiques constantes (coefficients techniques fixes du modèle input-output) et des élasticités - prix et - revenus dérivées du SLD (système à paramètres constants); on aurait aussi pu rendre dynamique le cadre en ce qui concerne le changement endogène des coefficients techniques (en fonction des prix relatifs et du temps, par ex.) ou des paramètres du SLD (avec des formulations cinématiques, par ex.). Mais comme il arrive souvent, nos sujets d'intérêts changèrent et le modèle tomba doucement dans l'oubli. 
Ces dernières années, on observe un rebondissement de l'intérêt pour la dynamique du changement technologique, d'abord à propos de la baisse de la croissance de la productivité dans la plupart des pays industriels avancés, constatée dans les années 1970 et 1980 et, plus récemment, par l'importance cette fois des augmentations de la croissance et de la productivité, principalement aux EtatsUnis, en raison, semble-t-il, de l'introduction massive des nouvelles technologies de l'information, ce qui a donné naissance au concept de Nouvelle Economie.

Le phénomène en question est typiquement mésoéconomique, c'est-à-dire que le comportement d'agents microéconomiques s'accompagne de changements structurels dans les relations au niveau technique, c'est-à-dire sectoriel, et que ces changements structurels modifient les conditions macroéconomiques de la croissance. Nous nous trouvons ainsi au coeur du fonctionnement opératoire dynamique de l'économie, c'est-à-dire au niveau de la mésoéconomie.

Dans une certaine mesure, il s'agit d'un problème similaire à celui que nous avons abordé: les contraintes de production conduisaient à des effets de transformation des consommations et de redistribution de surplus, en tous points identiques à ceux que peut provoquer un changement technologique qui modifie les fonctions d'offre.

Voyons donc ce que la mésoéconomie contemporaine a apporté comme éléments nouveaux pour traiter cette question.

Il conviendrait de situer le point de départ de cette réflexion dans un article pionnier de Baumol (1967) sur la croissance déséquilibrée. Dans cet article, d'une aveuglante simplicité, Baumol contemplait la dynamique d'une économie à deux secteurs: un secteur à productivité croissante et un secteur à productivité stagnante; dans une économie de marché avec une parfaite mobilité du travail, cette situation conduit automatiquement à une baisse du coût et des prix relatifs du premier secteur par rapport au deuxième, et s'il existe une possibilité de substitution entre les deux secteurs, le secteur à productivité stagnante finira par disparaître.

L'histoire nous rapporte aussi l'évidence que de nombreuses productions de biens et services où l'intervention humaine constituait le principal objet du produit et la productivité était donc nécessairement stagnante, (travail produit de l'artisan, du tailleur, du bottier, etc.) disparaissent pour faire place à des alternatives plus capitalisées où le travail humain est purement instrumental.

Toutefois, signalait Baumol, cette évolution pourrait très bien ne pas se produire, spécialement si les secteurs à productivité stagnante venaient à produire des biens et services très inélastiques au prix, où s'il se produisait des transferts de surplus venant subventionner ces activités (comme c'est le cas, par exemple, pour de nombreuses activités artistiques).

Si à l'extrême, signalait Baumol, la production réelle des deux secteurs devait se maintenir dans un rapport fixe, on assisterait alors à un profond changement de la structure de l'emploi en faveur des secteurs à productivité stagnante.

Cette dernière proposition du modèle de Baumol a fait l'objet d'une vérification empirique quelques années plus tard (Baumol, Batey Blackman, Wolf, 1985) qui a confirmé que, aux Etats-Unis, alors que les secteurs stagnants (mesurés en terme de taux de croissance de la productivité du travail directe ou systémique, ou encore de la productivité totale des facteurs) représentaient $31,5 \%$ de la production réelle et 32,4\% de l'emploi en 1947, ils atteignaient respectivement 28,9\% de la production et 43,0\% de l'emploi en 1976. 
Selon le modèle de Baumol, formalisé de manière plus complète en ce qui concerne les élasticités par Appelbaum, Schekatt (1994), ce résultat empirique est spécialement dû à des inélasticités élevées (par exemple pour de nombreux services personnels privés), ainsi qu'à des subventions (par exemple pour les services publics de marché).

Il se pourrait toutefois qu'une cause importante de l'évolution différenciée observée se situe au niveau des structures de marché des différents secteurs. En principe, les changements technologiques qu'implique l'évolution de la productivité totale des facteurs (PTF) devraient automatiquement se refléter dans les prix en concurrence parfaite. En d'autres termes, le taux de variation de la PTF devrait correspondre à l'inverse du taux de variation du prix relatif, et le produit des indices de prix relatifs et de la PTF relative devrait être unitaire.

Selon une vérification faite par Dalgaard (1989) et Fontela, Lo Cascio, Pulido (2000), il existe en effet une corrélation moyenne inverse satisfaisante entre prix et productivité, mais on constate aussi l'existence de nombreux secteurs où cette relation ne se trouve pas satisfaite. Parfois il s'agit de secteurs, tels que l'agriculture, dans lesquels les prix subissent des distorsions par rapport à un prix d'équilibre, en raison de politiques publiques. Parfois il s'agit de secteurs dans lesquels on constate de multiples situations de concurrence imparfaite.

Il convient donc d'analyser plus profondément la relation entre changement technique, structure des marchés et prix. Pour cela, le cadre input-output reste une méthodologie particulièrement attrayante. En effet, les coefficients techniques de Leonfief traduisent les structures de coût des secteurs économiques, et leur évolution dans le temps correspond à des changements technologiques; par ailleurs, la possibilité de calculer des prix relatifs avec le dual du modèle de Leontief correspond à la détermination de prix-coûts et, comme nous le verrons, permet aussi d'étudier l'impact sur les prix relatifs des structures de marché. La présentation la plus claire et didactique de cette utilisation de l'analyse input-output se trouve dans Carter (1990), et son traitement analytique dans Fontela (1989) et Fontela (1994).

Comme Baumol, Anne Carter partitionne le système économique en deux sous-systèmes, un dans lequel il se produit une modification des coefficients d'inputs correspondant à un gain de PTF (Peterson, 1979), et un ensemble d'autres secteurs représentant le reste de l'économie dans lequel il ne se produit aucun changement technologique. Utilisons son propre exemple avec quatre secteurs, un secteur $S$ producteur de matières premières et produits de base, un secteur $M$ producteur de produits manufacturés, un secteur $U$ de services de distribution et un secteur $R$ pour le reste de l'économie. Les données numériques de cet exemple, en forme de tableau input-output, sont les suivantes:

\begin{tabular}{|l|l|l|l|l|l|}
\hline & & $\mathrm{S}$ & $\mathrm{M}$ & $\mathrm{U}$ & \multicolumn{1}{l|}{$\mathrm{R}$} \\
\hline & $\mathrm{S}$ & 0 & 0.2 & 0 & 0 \\
& $\mathrm{M}$ & 0 & 0 & 0.5 & 0 \\
& $\mathrm{U}$ & 0 & 0 & 0 & 0.5 \\
& $\mathrm{R}$ & 0.5 & 0.1 & 0.2 & 0.1 \\
Travail $\ldots \ldots \ldots \ldots \ldots$ & $\mathrm{L}$ & 0.2 & 0.4 & 0.2 & 0.2 \\
Profit $\ldots \ldots \ldots \ldots \ldots$ & $\mathrm{P}$ & 0.3 & 0.3 & 0.1 & 0.2 \\
\hline & & 1.0 & 1.0 & 1.0 & 1.0 \\
\hline
\end{tabular}


Le système est en équilibre: la somme des coefficients techniques de chaque secteur plus les coefficients d'input primaires est unitaire et reflète donc les structures de coût. Les prix, égaux aux coûts, sont aussi unitaires.

Introduisons maintenant une innovation dans le secteur $M$ qui consiste simplement à éliminer l'utilisation de $R$ dans le processus de production.

La colonne $M$ devient donc:

\begin{tabular}{ll}
$\mathrm{S}$ & 0.2 \\
$\mathrm{M}$ & 0 \\
$\mathrm{U}$ & 0 \\
$R$ & 0 \\
$\mathrm{~L}$ & 0.4 \\
$\mathrm{P}$ & 0.3 \\
\hline & 0.9
\end{tabular}

Le coût a donc diminué de $10 \%$ et la question qui se pose est de savoir qui va être le bénéficiaire de ce gain de l'innovation, ou de ce surplus engendré par l'amélioration de la productivité totale des facteurs. Sur ce dernier point on constate que, après l'innovation, le secteur utilise 0.9 quantités d'inputs réels pour produire une unité, ce qui revient à dire que la productivité totale des facteurs (PTF) est devenue $1 / 0.9=1.11$, et a donc augmenté de $11 \%$, sans qu'il y ait eu pour autant augmentation de la productivité partielle du travail ou du capital, puisque dans cet exemple il s'agit uniquement d'une économie de facteurs intermédiaires.

Dans le cas où le secteur est en position structurelle de monopole, le gain ou surplus va être utilisé simplement pour augmenter le profit, qui deviendra alors 0.4 (au lieu de 0.3 ), ce qui ramènera le coût moyen à son niveau de départ unitaire. Tous les coûts étant unitaires, les prix restent unitaires, et il n'y a donc aucun changement pour les consommateurs. Ce sont les détenteurs du capital qui verront augmenter d'un tiers leurs revenus.

Par contre, dans le cas où le secteur $M$ est en situation de concurrence parfaite, le surplus va être distribué aux utilisateurs, et donc le prix va baisser de son niveau initial de 1 à celui de 0.9 .

Toutefois, les biens $M$ sont utilisés pour la production de $U$ au point qu'ils représentent 0.5 du coût unitaire de $U$; donc les coûts de $U$ vont baisser de 0.05 par suite de la baisse du prix de $M$, et si $U$ est aussi en concurrence parfaite, les prix de $U$ vont baisser à 0.95 . Et ainsi de suite. Comme Walras l'avait clairement mis en évidence, les prix sont interdépendants et la variation d'un prix entraîne des variations de tous les autres prix en situation d'équilibre général.

Pour calculer les effets directs et indirects des prix, on a donc recours au modèle dual des prix de Leontief qui conduit au résultat suivant:

$\begin{array}{ll}\text { Prix de } S & 0.984 \\ \text { Prix de } M & 0.895 \\ \text { Prix de } U & 0.94 \\ \text { Prix de } R & 0.967\end{array}$


Ainsi donc, la baisse initiale du prix de $U$ conduit à une baisse des autres prix, qui réduit les prix de ses propres inputs, et entraîne une baisse ultérieure de prix jusqu'à arriver au nouvel état d'équilibre avec un prix final de 0.895 pour $M$, qui est inférieur à la baisse initiale à 0.9. Attention toutefois : ce résultat ne se produit que si l'ensemble des secteurs évoluent en concurrence parfaite. Supposons, par exemple, que le secteur $S$, qui n'est pas en principe directement concerné par l'innovation de $M$, soit lui en position de monopole. Dans ce cas, même si les prix des biens que $S$ achète diminuent, on constate une augmentation des profits de ce secteur, alors que les prix restent constants. Et comme par ailleurs, dans cet exemple, $S$ est le seul fournisseur de $M$, la baisse du prix de $M$ ne peut pas se poursuivre audelà de la baisse initiale. Les nouveaux prix d'équilibre dans ce cas seront:

$\begin{array}{ll}\text { Prix de } S & 1.0 \\ \text { Prix de } M & 0.9 \\ \text { Prix de } U & 0.944 \\ \text { Prix de } R & 0.969\end{array}$

et le profit unitaire de $S$ augmentera du niveau initial de 0.3 à 0.316 .

Ce simple exemple numérique de Anne Carter suffit donc à montrer clairement que la répartition des gains de l'innovation est étroitement liée à la structure des marchés et qu'elle aboutit soit à une augmentation des profits (appropriation des bénéfices de l'innovation sous forme de revenus par les détenteurs du capital), soit à une baisse de prix (distribution des bénéfices de l'innovation aux consommateurs sous forme d'une augmentation du pouvoir d'achat d'origine).

Dans un travail parallèle mais qui utilisait l'approche française de l'analyse des gains de la croissance (Massé et Bernard, 1969; Courbis et Templé, 1975), nous avions montré (Fontela, 1989) que le processus de répartition des surplus dérivés d'une innovation, c'est-à-dire d'un accroissement de la productivité totale des facteurs traduisait, en fait, la position de tous les agents économiques en termes de rapports de force, et que les travailleurs et leurs syndicats, par delà l'hypothèse de marchés du travail en concurrence parfaite, intervenaient aussi dans le processus, affectant ainsi la distribution fonctionnelle et personnelle du revenu, qui avait été analysée par Camilo Dagum lors de la Conférence Solari de 1996 (Dagum, Fontela, 1997 et Dagum, 1999).

On parvenait ainsi à l'équation de répartition des surplus de PTF

$$
P T F=\left(S^{\prime} i-S i\right)+s_{l}+s_{k}-s_{f}
$$

dans laquelle la matrice $S$ décrit les flux de gains de PTF entre agents de la production qui découlent des variations de prix des inputs, les vecteurs $s_{l}$ et $s_{k}$ établissent les gains qui reviennent aux travailleurs et aux détenteurs du capital et le vecteur $s_{f}$ ceux qui reviennent aux consommateurs lorsque les prix des biens qu'ils achètent diminuent. Cette expression de la répartition des surplus est directement utilisable pour de nombreuses applications numériques tant au niveau microéconomique des entreprises, qu'au niveau mésoéconomique des secteurs ou des régions ou au niveau macroéconomique des nations (Fontela, Pulido 1993).

Ainsi à titre illustratif, on peut présenter l'exemple d'une grande entreprise suisse de distribution, Migros, calculé par Antille, Fontela (1988) à partir d'infor- 
mations sur le compte d'exploitation à prix courants et à prix constants de cette entreprise. Les calculs effectués montrent pour l'année 1985 un gain de productivité totale des facteurs de 80 millions de Francs.

\section{Répartition du «surplus» de la Communauté Migros en 1985}

(en millions de francs)

\begin{tabular}{|l|r|}
\hline Origine: & \\
\hline - Surplus de productivité & 80 \\
- Héritage dus à la baisse du prix des matières premières & 187 \\
\hline Total & 267 \\
\hline Répartition: & \\
\hline - Aux consommateurs & 120 \\
- A l'agriculture et autres fournisseurs & 93 \\
- Aux salariés & 30 \\
- Aux administrations & - \\
- Aux détenteurs de capital & 24 \\
\hline Total & 267 \\
\hline
\end{tabular}

Dans l'équation 8, interviennent à la fois les consommateurs, les salariés et les détenteurs de capital, ainsi que les fournisseurs, avec une claire distinction dans le cas de Migros entre fournisseurs de matières premières dont le prix d'achat pour Migros a baissé en 1985, et d'autres fournisseurs suisses, tels que les producteurs agricoles, dont le prix a, par contre, augmenté.

Si l'on considère que les consommateurs bénéficient à la fois des surplus de productivité et du solde de ces transferts envers les fournisseurs, on aboutit à la répartition nette suivante du gain de productivité de Migros (80 millions).

$$
\begin{array}{ll}
\text { - aux consommateurs } & 26 \text { millions } \\
\text { - aux salaires } & 30 \text { millions } \\
\text { - aux détenteurs du capital } & 24 \text { millions }
\end{array}
$$

On voit donc que ce schéma de répartition répond à d'autres critères que la simple pression de la concurrence sur le niveau des prix, et dans le cas de Migros, par ex., il tient compte des caractéristiques spécifiques du mouvement coopératif.

Ce que cet ensemble de travaux microéconomiques, comme celui que nous venons de voir, mésoéconomiques ou macroéconomiques a mis en évidence, c'est le processus par lequel un choc exogène, par exemple une innovation technologique, a des effets divers sur les prix et sur les revenus; ce que l'on ne sait par contre pas clairement, c'est dans quelle mesure ces effets sont plus ou moins dynamiques.

Lorsqu'il y a un gain de productivité, est-il plus avantageux pour la croissance à long terme, qu'il se traduise par une baisse généralisée des prix, par une hausse généralisée des revenus, ou existe-t-il une combinaison optimale entre les deux?

A vrai dire, les économistes ne savent pas encore grand chose sur ce type de dynamique mésoéconomique, si ce n'est qu'elle se relie en termes macroécono- 
miques au modèle de croissance déséquilibrée de Baumol, et qu'elle repose sur la microéconomie de la concurrence imparfaite et des relations de pouvoir dans les marchés.

Revenons au modèle FSD initial:

- d'un côté l'analyse de contraintes de production traduisait l'existence de pouvoirs de l'offre, c'est-à-dire de situations de concurrence imparfaite;

- d'un autre côté, l'utilisation des modèles input-output établissait clairement le fonctionnement de l'interdépendance, les coefficients techniques et les coefficients de valeur ajoutée décrivant la position des partenaires économiques du jeu de pouvoir, tels que les fournisseurs de matières premières et produits intermédiaires, les travailleurs et les détenteurs du capital;

- finalement le système linéaire de dépenses, SLD, ici aussi avec ses nombreuses limitations, définissait les élasticités et revenus des consommateurs, et donc les fondements de leur pouvoir sur le marché.

Alors que dans l'exemple de Anne Carter, ou dans le modèle de répartition du surplus, on se limite soit à une analyse de statique comparative, soit à une évaluation du passé, avec l'inclusion des fonctions de demande en liaison avec le modèle de production, on aurait pu aller un pas plus loin dans la mesure où l'on utilise un instrument qui aurait pu même donner des réponses dynamiques, si l'on était parvenu à expliquer le processus de changement des coefficients (ce qui d'ailleurs, ne semblait pas être totalement impossible à l'époque).

Interprétons donc pour finir le mécanisme de la Nouvelle Economie contemporaine à la lumière de ce qui vient d'être dit. La Nouvelle Economie est un concept qui a été développé aux Etats-Unis pour expliquer le modèle de croissance de la fin du $\mathrm{XX}^{\mathrm{e}}$ siècle, modèle caractérisé sur le plan macroéconomique par une croissance rapide permettant d'atteindre le niveau de plein emploi sans provoquer des pressions inflationnistes, et en utilisant pour cela une politique budgétaire restrictive et une politique monétaire tout aussi restrictive. On se trouve en fait dans une situation qui est l'envers de celle de la stagflation de la fin des années soixante-dix et des années quatre-vingts, lorsque la croissance était très faible et le chômage augmentait dans un contexte inflationniste et de politiques budgétaires et monétaires expansives.

A la base de cette croissance de la Nouvelle Economie américaine, on retrouve certainement un retournement du processus de croissance de la productivité totale des facteurs, dont on observe une accélération au cours des dix dernières années. Cette croissance de la PTF s'appuie sur un système accru d'innovation qui probablement peut-être associé à l'introduction massive des technologies du nouveau paradigme de la Société de l'Information (ordinateurs, microélectronique et télécommunications) dans tous les procédés et les produits. Si c'est le cas, nous nous trouvons devant un important choc technologique dont les mécanismes de diffusion doivent suivre les chemins que nous avons décrits précédemment.

D'un côté l'innovation en régime concurrentiel doit amener une réduction des prix. Aux Etats-Unis (et ailleurs), on constate en effet une chute rapide des prix des équipements caractéristiques de la Société de l'Information - ordinateurs, terminaux, microcircuits, etc. - , des biens et services qui leur sont directement 
associés - informatique, télécommunications, robotisation, etc. et en général de tous les biens et services qui incorporent ces nouvelles technologies. Le processus a sans doute été accéléré par l'adoption de politiques de démantèlement des monopoles publics (télécommunications) ou par l'application d'une politique active de soutien de la concurrence sur les marchés (cas des ordinateurs, par ex.). En parallèle, l'innovation a permis une augmentation de la rénumération des travailleurs spécialisés de haut niveau de qualification dans les secteurs innovateurs (par ex. les informaticiens) et une plus grande rémunération du capital dans les cas où l'innovation technologique a fourni un monopole de courte durée sur les nouveaux produits ou procédés (par ex. dans les industries dites de haute technologie). Cet effet primaire de l'innovation sur les prix et les revenus étant établi, il convient alors d'examiner ses conséquences sur la dynamique de la consommation.

On assistera à une augmentation du revenu réel des ménages avec des effets redistributifs importants. C'est clair que les travailleurs qui parviennent par leur rareté à obtenir une partie du surplus, ou les détenteurs de capital, ne constituent qu'une partie de la population (même si le capitalisme populaire a fait des grands progrès dans l'économie américaine), et donc le modèle qui va suivre va être nécessairement amplificateur des écarts existants entre riches et pauvres.

L'effet dynamique sur la croissance est toutefois de nature plus complexe, et se réfère aux caractéristiques des fonctions de consommation et des élasticités prix et revenu qui leur sont associées. Si les nouveaux biens et services ont un caractère supérieur, c'est-à-dire qu'ils ont des élasticités prix et revenus élevées, le double effet de la baisse des prix relatifs de ces nouveaux produits et de l'augmentation des revenus des ménages les plus riches, entraînera une croissance explosive de la demande.

Nous nous retrouverons alors dans un véritable cercle vertueux: l'augmentation de la demande stimulera l'introduction ultérieure d'innovations, qui à leur tour abaisseront les prix et augmenteront les revenus conduisant à nouveau à des augmentations de la demande. C'est cela dans le fond le cercle vertueux de la Nouvelle Economie, un processus mésoéconomique dont l'ampleur est telle qu'il permet une croissance rapide de l'économie et de l'emploi tout en évitant des pressions à la hausse des prix.

Mais la croissance n'est pas aussi facile que le cercle vertueux le laisse entendre. Ce même cercle vertueux a déjà été le moteur d'autres longues périodes d'expansion de l'économie mondiale, évidemment avec des moyens d'innovation autres que ceux de la Société de l'Information. C'est ainsi que dans l'après-guerre européenne, la soif de biens durables (automobiles, équipements ménagers, logements) rendit très élastiques aux prix et aux revenus ces biens qui bénéficièrent en particulier des gains d'innovations associés tout spécialement aux économies d'échelle. Et il est bon de rappeler que ce modèle cessa de promouvoir un cercle vertueux lorsque les parcs de biens durables se rapprochèrent des niveaux de saturation.

Aujourd'hui cette échéance peut paraître éloignée, telle est la soif de PC, de téléphones mobiles ou de services d'Internet, et leur expansion épidémiologique, mais c'est évident que le maintien à long terme du fonctionnement du cercle vertueux requiert un flux continu d'innovations ayant pour caractéristique essentielle la production de biens et services supérieurs. Pour reprendre la terminologie du 
SLD, si chère à Solari, on peut considérer que le cercle vertueux se maintiendra tant que les nouvelles technologies permettront d'introduire sur le marché des produits avec un coefficient $\gamma$ (de consommation obligée) encore bas, et un coefficient budgétaire $\beta$ d'utilisation du revenu discrétionnaire, encore relativement élevé.

Qui plus est, le succès à long terme du cercle vertueux de la Nouvelle Economie pose d'autres problèmes qu'on ne peut négliger:

- les entreprises doivent être capables d'extraire de la tourmente technologique les informations nécessaires pour maintenir le flux continu d'innovations, et doivent pouvoir avoir accès aux facteurs de production nécessaires: des lignes prometteuses peuvent dépérir par manque de financement ou de travail adapté aux besoins, d'où la nécessité d'une grande flexibilité des marchés des facteurs primaires;

- les systèmes financiers doivent être capables d'effectuer des choix positifs sur la base de rendements escomptés, alors qu'on sait que l'information est asymétrique, et que les plans des entreprises ne sont pas toujours représentatifs de futurs possibles;

- et les travailleurs doivent trouver dans des systèmes de formation la possibilité d'augmenter les perspectives d'emploi, au risque de tomber, par suite du processus innovateur schumpeterien de destruction créatrice, en situation d'obsolescence totale.

La croissance dans la Nouvelle Economie est une croissance fortement déséquilibrée au sens de Baumol avec des secteurs dont les productivités augmentent très rapidement, et d'autres dans lesquels elles augmentent lentement, stagnent voire décroissent.

La régulation de ce système sur le plan social, financier ou économique, est très complexe et réclame de nouvelles formes de gouvernance. Je n'ai aucun doute qu'avec la Nouvelle Economie les risques de fractures sociales, de crises financières, voire même de crises globales du système économique sont en train d'augmenter rapidement et que des politiques actives sont nécessaires pour les éviter. Le marché est un système efficace de résolution de problèmes microéconomiques, mais il ne peut répondre seul aux problèmes que pose la mésoéconomie contemporaine. C'est à cette régulation nécessaire que Solari, Duval et moi-même nous nous référions dans notre travail de 1971, lorsque nous concluions qu'un système d'impôts indirects discriminant sectoriellement pouvait augmenter le niveau de bien-être en cas d'apparition de contraintes de production. De la même manière, nous réclamerions aujourd'hui un nouveau système fiscal pour la Nouvelle Economie, qu'il n'est pas possible d'analyser ici.

Le tour de la question a été fait. On peut imaginer aujourd'hui des circonstances dans lesquelles le modèle FSD aurait encore un sens, et on saurait aussi comment le compléter et l'améliorer pour augmenter sa portée opératoire dans le sens de son utilisation dans une démarche d'expérimentation. Nous entendons par expérimentation, en un sens très large, toute action directe ou indirecte effectuée sur une réalité donnée en vue de susciter ou de recueillir des conséquences observables (Solari, 1963, p. 285). 
Il reste, pour conclure ce dialogue avec Solari d'essayer de déceler quelle aurait pu être l'évolution de sa pensée en fonction des nouvelles préoccupations des économistes.

Je crois qu'avec son intérêt pour la désagrégation des phénomènes de production et de consommation, Solari s'inscrirait clairement dans le courant, encore aujourd'hui embryonnaire, de la mésoéconomie. Toujours respectueux d'une vision européenne de la science économique qui recherche une interaction permanente entre théorie et réalité et qui incorpore raisonnement et expérimentation, il aurait inclus dans ses enseignements de nouveaux instruments dont l'utilisation se situe à ce niveau mésoéconomique, tels que les modèles d'équilibre général calculable.

Et je suis convaincu qu'il aurait été tout spécialement attiré par les versions dynamiques de ces modèles d'équilibre général calculables, en liaison avec les modèles multisectoriels d'exploration. En fait, comme le disait Leontief (1941) avec perspicacité, c'est dans cette liaison que se situe le défi le plus attrayant pour la recherche économique, un défi dont l'importance et la complexité ne font qu'augmenter chaque jour.

\section{Universidad Autónoma de Madrid}

\section{RÉFÉRENCES}

Antille, G., (1978), «Etude et comparaison de modèles de prix avec application au cas de la Suisse.» Collection des thèses, Berne, Peter Lang.

Antille, G., Fontela, E., (1988), «Origine et distribution de la productivité globale», Revue Suisse de Statistique et Economie Politique, 3, p. 277-287.

Appelbaum, E., Schettkat, R., (1994), «The End of Full Employment? On Economic Development in Industrialized Countries », Intereconomics, mai-juin, pp. 122-130.

Baranzini, E., (1978), «L'analyse des modèles d'entrées-sorties en économie. Problèmes de méthodes et pratiques opératoires.» Collection des thèses, Berne, Peter Lang.

Baumol, W.J., (1967), «Macroeconomics of Unbalanced Growth: The Anatomy of Urban Crisis», American Economic Review, 57, 415-26.

Baumol, W.J., Batey Blackman S.A., Wolff, E.N., (1985), «Unbalanced Growth Revisited: Asymptotic Stagnancy and New Evidence», American Economic Review, 75, 806-816.

Carlevaro, F., (1975), «Sur la comparaison et la généralisation de certains systèmes de fonctions de consommation semi-agrégée », Collection des thèses, Berne, Peter Lang.

Carter, A.P., (1990), «Upstream and Downstream Benefits of Innovation», Economic Systems Research, 2, 241-257.

Courbis, R., Templé, P., (1975), «La méthode des comptes de surplus et ses applications macroéconomiques », INSEE série C, 35, Paris.

Dalgaard, E., (1989), «System Productivity Time Series for Denmark 1966-85», Contribution à la 9ème Conférence Internationale sur les Techniques Input-Output, Kesztheky, Hongrie.

Dagum, C., (1999), «A Study on the distributions of Income, Wealth, and Human Capital », Conférence Solari de 1996, Revue européenne des sciences sociales, Tome XXXVII, No 113, pp. 231-268.

Dagum, C., Fontela, E., (1997), «Partage des gains de productivité et distribution fonctionnelle du Revenu », Communication à l'Association Internationale des Economistes de Langue Française, Porto. 
Fontela, E., Solari, L., Duval, A., (1971), «Contraintes de production, prix et valeurs ajoutées dans un contexte input-output», Publications Econométriques, vol. IV, 2, Sirey, Paris.

Fontela, E., Solari, L., Duval, A., (1972), «Production constraints and prices in an input-output system », in A. Brody, A.P. Carter (eds), Input-Output Techniques, North-Holland Publishing.

Fontela, E., (1989), «Industrial Structures and Economic Growth: an Input-Ouptut Perspective», Economic Systems Research, Vol. 1, No 1, pp. 45-52.

Fontela, E., Pulido, A., (1993), «Analisis Input-Output, Modelos, Datos y Aplicaciones », Piramide, Madrid.

Fontela, E., (1994), «Inter-industry Distribution of Productivity Gains », Economic Systems Research, Vol. 6, No 3, pp. 227-236.

Fontela, E., Lo Cascio, M., Pulido, A., (2000), «Systemic Productivity and Relative Prices in an InputOutput Framework», Contribution à la 13ème Conférence Internationale sur les Techniques Input-Output, Macerata, Italie.

Garau, G., (1996), «La répartition des gains de la croissance: une analyse entrée-sortie.» Publications universitaires européennes, Série 5, Sciences économiques et politiques; vol. 2034.

Juillard, M., (1993), «Un schéma de reproduction pour l'économie des Etats-Unis: 1948-1980. Tentative de modélisation et quantification.»Collection des thèses, Berne, Peter Lang.

Leontief, W., (1941), «The Structure of the American Economy», Oxford Univ. Press, New York.

McNeill, G., (1978), «Contribution à l'analyse des modèles multisectoriels. Analyses de la fonction de production de Wigley et de son introduction dans un modèle sectoriel d'équilibre statique.»Collection des thèses, Berne, Peter Lang.

Massé, P., Bernard, P., (1969), «Les Dividendes du Progrès », Seuil, Paris.

Peterson, W., (1979), «Total Factor Productivity in the UK: a Disaggregated Analysis», in Patterson, Schott (eds.), The Measurement of Capital, Macmillan.

Rossier, E., (1974), «Contribution aux explications dynamiques de la consommation semi-agrégée.» Collection des thèses, Berne, Peter Lang.

Snella, J.-J., (1976), «Théorie des choix et approches empiriques dans l'étude de la consommation privée. Analyse et expériences.» Collection des thèses, Berne, Peter Lang.

Solari, L., (1963), «Modèles et Décisions Economiques », Essai sur les fondements de l'économie pure, Genève, Droz.

Solari, L., (1971), «Théorie des choix et fonctions de consommation semi-agrégées, Modèles statistiques », Genève-Paris, Droz.

Stone, R., (1961), «Input-output and national accounts », OECD, Paris. 